\title{
The smallest fullerene without a spiral
}

\author{
Gunnar Brinkmann ${ }^{\mathrm{a}, *}$, Jan Goedgebeur ${ }^{\mathrm{a}}$, Brendan D. McKay ${ }^{\mathrm{b}}$ \\ ${ }^{a}$ Applied Mathematics \& Computer Science, Ghent University, Krijgslaan 281-S9, 9000 Ghent, Belgium \\ ${ }^{\mathrm{b}}$ Research School of Computer Science, Australian National University, ACT 0200, Australia
}

\section{A R T I C L E I N F O}

\section{Article history:}

Received 8 November 2011

In final form 22 November 2011

Available online 29 November 2011

\begin{abstract}
A B S T R A C T
In this note, we give the result of a computer search for the smallest fullerene that does not allow a face spiral code as used by Manolopoulos and Fowler and adopted in IUPAC recommendations for fullerene nomenclature. The search enumerated all the small fullerenes on up to 400 vertices and the conclusion is that the smallest fullerene without a face spiral has 380 vertices.
\end{abstract}

(c) 2011 Elsevier B.V. All rights reserved.
The first algorithm to construct lists of fullerenes was the spiral algorithm of Manolopoulos and Fowler [1,2]. It constructs fullerenes by enumerating encodings of face spirals. A clockwise (resp. counterclockwise) face spiral of a fullerene with $k$ faces is a sequence of distinct faces $\left(f_{1}, f_{2}, \ldots, f_{k}\right)$ with the property that $f_{1}$ and $f_{2}$ share an edge and that for $3 \leqslant i \leqslant k$ face $f_{i}$ has a connected intersection with $\left\{f_{1} \cup f_{2} \cup \ldots \cup f_{i-1}\right\}$ and shares an edge $e$ with $f_{i-1}$ that is the last of those edges of $f_{i-1}$ in clockwise (resp. counterclockwise) orientation around $f_{i-1}$ that belong to no face in $\left\{f_{1}, f_{2}, \ldots, f_{i-2}\right\}$. For fullerenes such face spirals can be encoded as sequences of $5 \mathrm{~s}$ and 6 s giving the face sizes, or more compactly as a sequence of length 12 giving the position of the 12 pentagons in the spiral.

Already in [3] Manolopoulos and Fowler gave an example of a fullerene with 380 vertices that does not allow any face spirals and therefore would be missed by the algorithm. The question was whether this is the smallest counterexample. In [4] it is shown that in the more general class of all cubic polyhedra, the smallest counterexample has only 18 vertices, so one might have expected a smaller counterexample also in the class of fullerenes. For efficiency reasons, when applying the spiral algorithm for the generation of fullerenes, Manolopoulos and Fowler restricted the generation to spirals starting with a pentagon (see [2]). It is known that the smallest fullerene that is missed in this way is one isomer of $C_{100}$. Tables 1 gives the numbers of fullerenes up to 400 vertices that do not allow spirals starting at a pentagon. The structures can be downloaded from http://hog.grinvin.org/Fullerenes.

After a much more efficient and also complete algorithm for fullerene enumeration was developed [5], there were no longer algorithmic reasons to ask for the smallest fullerenes without spirals, but as an IUPAC proposal recommended the use of face spirals as a basis for fullerene nomenclature [6], it is still important to know the smallest fullerenes to which such a nomenclature could not be applied.

In [7] a new and faster algorithm using a recursive structure for the class of fullerenes [8] was developed that could generate and test all fullerenes up to 400 vertices. The result is that the fullerene with 380 vertices depicted in Figure 1 is the smallest fullerene without a spiral. This fullerene was already given in [3] but at that time minimality could not be proven. For computational results independent checks are very important to minimize the chance of errors, and so we independently confirmed this minimality result with the program from [5]. In the range from 382 to 400 vertices, fullerenes were generated and tested by the first program alone. In this range, the fullerene with 384 vertices depicted in Figure 2, is the only fullerene without a spiral. Again, this example was obtained earlier by construction (in [9]).

\footnotetext{
* Corresponding author.

E-mail addresses: Gunnar.Brinkmann@UGent.be (G. Brinkmann), Jan.Goedgebeur@UGent.be (J. Goedgebeur), bdm@cs.anu.edu.au (B.D. McKay).
} 
Table 1

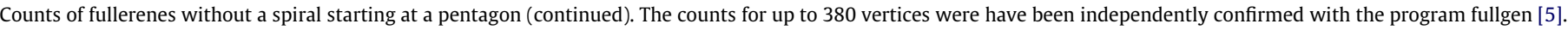

\begin{tabular}{|c|c|c|c|c|c|c|c|c|c|c|c|}
\hline Vertices & Fullerenes & Vertices & Fullerenes & Vertices & Fullerenes & Vertices & Fullerenes & Vertices & Fullerenes & Vertices & Fullerenes \\
\hline 100 & 1 & 150 & 0 & 200 & 36 & 250 & 411 & 300 & 5548 & 350 & 30885 \\
\hline 102 & 0 & 152 & 3 & 202 & 25 & 252 & 615 & 302 & 4388 & 352 & 41747 \\
\hline 104 & 1 & 154 & 0 & 204 & 59 & 254 & 467 & 304 & 6193 & 354 & 35180 \\
\hline 106 & 0 & 156 & 3 & 206 & 35 & 256 & 851 & 306 & 4938 & 356 & 47021 \\
\hline 108 & 0 & 158 & 0 & 208 & 66 & 258 & 562 & 308 & 7673 & 358 & 39661 \\
\hline 110 & 1 & 160 & 1 & 210 & 37 & 260 & 881 & 310 & 6242 & 360 & 51978 \\
\hline 112 & 0 & 162 & 1 & 212 & 89 & 262 & 623 & 312 & 9165 & 362 & 44499 \\
\hline 114 & 0 & 164 & 10 & 214 & 57 & 264 & 1083 & 314 & 7261 & 364 & 57767 \\
\hline 116 & 0 & 166 & 3 & 216 & 85 & 266 & 863 & 316 & 10302 & 366 & 50370 \\
\hline 118 & 0 & 168 & 10 & 218 & 62 & 268 & 1270 & 318 & 8464 & 368 & 66261 \\
\hline 120 & 0 & 170 & 4 & 220 & 87 & 270 & 1037 & 320 & 11854 & 370 & 58003 \\
\hline 122 & 0 & 172 & 8 & 222 & 64 & 272 & 1558 & 322 & 9745 & 372 & 77534 \\
\hline 124 & 1 & 174 & 2 & 224 & 172 & 274 & 1133 & 324 & 14356 & 374 & 68670 \\
\hline 126 & 0 & 176 & 14 & 226 & 99 & 276 & 1968 & 326 & 12344 & 376 & 89284 \\
\hline 128 & 0 & 178 & 6 & 228 & 198 & 278 & 1525 & 328 & 17926 & 378 & 77802 \\
\hline 130 & 0 & 180 & 8 & 230 & 141 & 280 & 2529 & 330 & 15397 & 380 & 100355 \\
\hline 132 & 1 & 182 & 9 & 232 & 194 & 282 & 2002 & 332 & 21182 & 382 & 86960 \\
\hline 134 & 1 & 184 & 16 & 234 & 141 & 284 & 3011 & 334 & 17986 & 384 & 112914 \\
\hline 136 & 1 & 186 & 10 & 236 & 316 & 286 & 2473 & 336 & 23625 & 386 & 101046 \\
\hline 138 & 0 & 188 & 18 & 238 & 205 & 288 & 3413 & 338 & 19571 & 388 & 131212 \\
\hline 140 & 1 & 190 & 6 & 240 & 400 & 290 & 2783 & 340 & 26885 & 390 & 117963 \\
\hline 142 & 0 & 192 & 33 & 242 & 259 & 292 & 4215 & 342 & 22801 & 392 & 152483 \\
\hline 144 & 2 & 194 & 13 & 244 & 468 & 294 & 3401 & 344 & 31476 & 394 & 134408 \\
\hline 146 & 0 & 196 & 34 & 246 & 397 & 296 & 4996 & 346 & 26842 & 396 & 171302 \\
\hline \multirow[t]{2}{*}{148} & 2 & 198 & 18 & 248 & 634 & 298 & 3797 & 348 & 35834 & 398 & 150285 \\
\hline & & & & & & & & & & 400 & 189662 \\
\hline
\end{tabular}

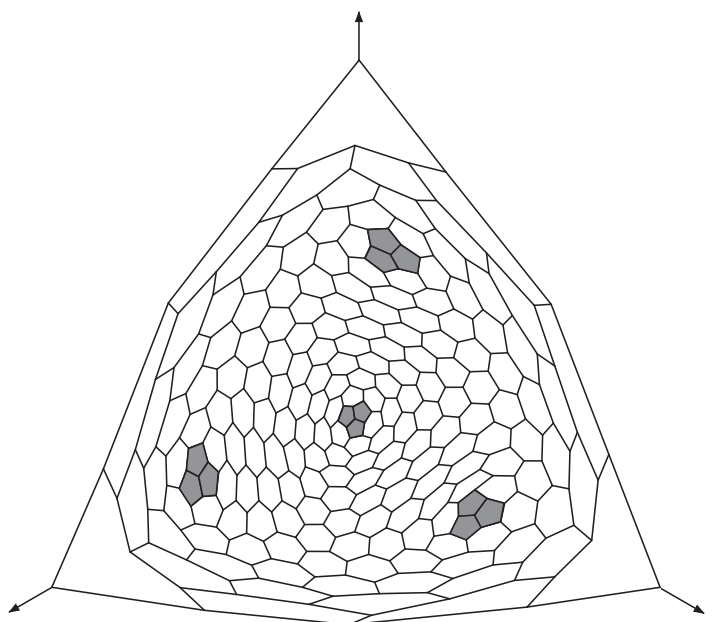

Figure 1. The smallest fullerene without a spiral. It has 380 vertices and was first described in [3]. In order to show the rotational symmetry with vertices as centres of rotation, one vertex has to be chosen at infinity. The fullerene has chiral tetrahedral symmetry.

\section{Acknowledgments}

This work was carried out using the Stevin Supercomputer Infrastructure at Ghent University. Jan Goedgebeur is supported by a Ph.D. Grant from the Research Foundation of Flanders (FWO). Brendan McKay is supported by the Australian Research Council.

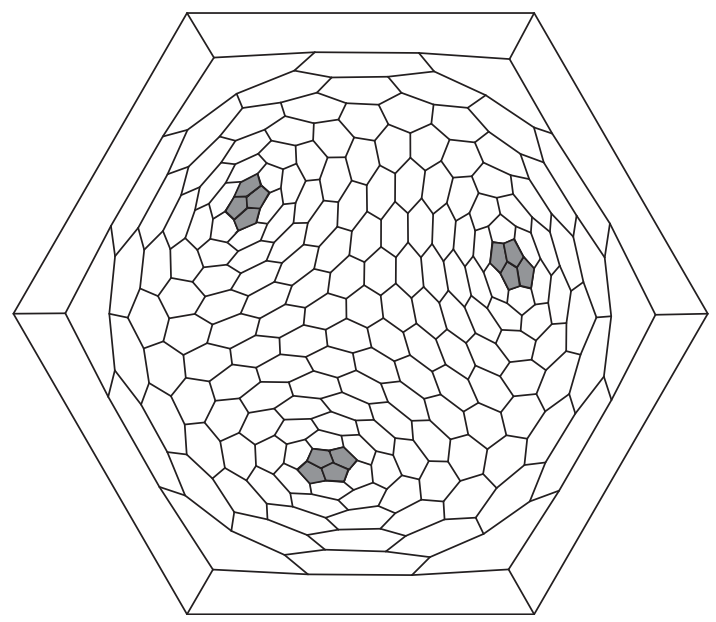

Figure 2. The second smallest fullerene without a spiral. It has 384 vertices and was first described in [9]. The fullerene has $D_{3}$ symmetry.

\section{References}

[1] D.E. Manolopoulos, J.C. May, S.E. Down, Chem. Phys. Lett. 181 (2,3)(1991)105-111.

[2] P.W. Fowler, D.E. Manolopoulos, An Atlas of Fullerenes, Oxford University Press, 1995.

[3] D.E. Manolopoulos, P.W. Fowler, Chem. Phys. Lett. 204 (1-2) (1993) 1-7.

[4] G. Brinkmann, Chem. Phys. Lett. 272 (3-4) (1997) 193-198.

[5] G. Brinkmann, A.W.M. Dress, J. Algor. 23 (1997) 345-358.

[6] E.W. Godly, R. Taylor, Pure Appl. Chem. 69 (7) (1997) 1411-1434.

[7] G. Brinkmann, J. Goedgebeur, B.D. McKay (in preparation).

[8] M. Hasheminezhad, H. Fleischner, B.D. McKay, Chem. Phys. Lett. 464 (2008) 118-121.

[9] M. Yoshida, P.W. Fowler, J. Chem. Soc., Faraday Trans. 93 (18) (1997) 32893294. 